\title{
Erratum: Transitive Separation Logic
}

\author{
Han-Hing Dang and Bernhard Möller \\ Institut für Informatik, Universität Augsburg, D-86159 Augsburg, Germany \\ \{h. dang, moeller\} ainformatik.uni-augsburg.de
}

W. Kahl and T.G. Griffin (Eds.): RAMiCS 2012, LNCS 7560, pp. 1-16, 2012.

(C) Springer-Verlag Berlin Heidelberg 2012

DOI 10.1007/978-3-642-33314-9_24

Definition 5.4 (p. 8):

- In item 1. add: As an additional case we also consider $\square$ to be a linked structure.

- In item 2. the first two sentences should read as follows:

$a$ is a forest if $a$ is acyclic and has maximal in-degree 1 except possibly for $\square$ nodes. Algebraically this is expressed by the dual of the formula for determinacy, namely $\forall p:\left|a^{\prime}\right\rangle\left\langle a^{\prime}\right| p \leq p$, where $a^{\prime}={ }_{d f} a \cdot \neg \square$.

Definition 5.7 (p. 9) should read as follows:

For identifier i, selector name $l$ and expression $e$ we set

$$
\text { i. } l:=e=_{d f} \quad\left\{\left((s, a),\left(s,\left(s(\mathrm{i}) \mapsto \llbracket e \rrbracket_{(s, a)}\right) \mid a_{l}\right): s(\mathrm{i}) \neq \square, s(\mathrm{i}) \leq\ulcorner a\},\right.\right.
$$

where the update $\mid a_{l}$ leaves all $a_{k}$ with $k \neq l$ unchanged.

Definition 6.1 (p. 9): The definition of links can be discarded.

Definition 6.4 (p. 9) should read as follows:

For trees $a_{1}, a_{2}$ we define directed combinability by

$$
a_{1} \triangleright a_{2} \quad \Leftrightarrow_{d f} \quad\left\ulcorner a_{1} \cdot \overparen{a_{2}}=0 \wedge a_{1} \cdot a_{2} \leq \square \wedge a_{1}\right\urcorner \cdot a_{2}=\operatorname{root}\left(a_{2}\right) .
$$

Lemma 6.5 (p. 10) should read as follows:

If $a$ is a tree then $\square \triangleright a \Leftrightarrow$ FALSE and $a \triangleright \square \Leftrightarrow \square \leq a$. 
Lemma 6.6 (p. 10) should read as follows:

For trees $a_{1}$ and $a_{2}$, assume $a_{1} \triangleright a_{2}$. Then $\operatorname{terms}\left(a_{1}+a_{2}\right)=\left(\operatorname{terms}\left(a_{1}\right)-\right.$ $\left.\operatorname{root}\left(a_{2}\right)\right)+\operatorname{terms}\left(a_{2}\right)$ and $\operatorname{root}\left(a_{1}+a_{2}\right)=\operatorname{root}\left(a_{1}\right)$.

In the proof of Lemma 6.6, the part concerning the formula for root should read as follows:

For root we first observe that $a_{1} \neq \square$ by Lemma 6.5. Next, we calculate, symmetrically, $\left.\left.\operatorname{root}\left(a_{1}+a_{2}\right)=\left\ulcorner a_{1} \cdot \neg a_{1}\right\urcorner \cdot \neg a_{2}\right\urcorner\left\ulcorner a_{2} \cdot \neg a_{1}\right\urcorner \cdot \neg a_{2}\right\urcorner$.

The first summand reduces to $\left\ulcorner a_{1} \cdot \neg a_{1}^{\urcorner}=\operatorname{root}\left(a_{1}\right)\right.$, since $a_{1} \triangleright a_{2}$ implies $\left\ulcorner a_{1} \cdot a_{2}\right\urcorner=0$, i.e., $\left\ulcorner a_{1} \leq \neg a_{2}\right\urcorner$. The second summand is, by definition, equal to $\left.\operatorname{root}\left(a_{2}\right) \cdot \neg a_{1}\right\urcorner$. Since $a_{1} \triangleright a_{2}$ implies $\left.\operatorname{root}\left(a_{2}\right) \leq a_{1}\right\urcorner$, this summand reduces to 0 .

Page 11 The definitions of list and I_cell should read as follows:

$$
\begin{aligned}
\text { list } & =d f\{a: a \text { is a chain }\}, \\
\text { I_cell } & =d f\{a: a \text { is a cell }\} .
\end{aligned}
$$

Page 11 In the proof of Lemma 7.4 the third and fourth paragraphs should read as follows:

Finally, we prove $\left.a_{2}\right\urcorner \cdot r_{3}=\operatorname{root}\left(a_{3}\right)$ and hence $a_{2}+a_{3} \in P_{2} \bowtie P_{3}$. First, one can show that for deterministic $a$ and arbitrary $b$ one has $a \triangleright b \Rightarrow \operatorname{root}(b)=$ terms $(a)$. Set now $r_{i}={ }_{d f} \operatorname{root}\left(a_{i}\right)$. By $a_{12} \triangleright a_{3}$ we have $\left.r_{3}=a_{12}\right\urcorner \cdot\left\ulcorner a_{3}=\left(a_{1}\right\urcorner+\right.$ $\left.\left.a_{2}\right\urcorner\right) \cdot\left\ulcorner a_{3}=a_{1}\right\urcorner \cdot\left\ulcorner a_{3}+a_{2}\right\urcorner \cdot\left\ulcorner a_{3}\right.$. Since $r_{3}$ is atomic and $\left.\left.a_{1}\right\urcorner \cdot a_{2}\right\urcorner=0$ by $a_{1} \triangleright a_{2}$, it follows that $\left.r_{3}=a_{1}\right\urcorner \cdot\left\ulcorner a_{3}\right.$ or $\left.r_{3}=a_{2}\right\urcorner \cdot a_{3}$. Assume $\left.r_{3}=a_{1}\right\urcorner \cdot\left\ulcorner a_{3}\right.$. Then terms $\left(a_{1}\right)=r_{3}$ in contradiction to terms $\left(a_{1}\right)=r_{2}$ by $a_{1} \triangleright a_{2}$ and $\left\ulcorner a_{2} \cdot\left\ulcorner a_{3}=0\right.\right.$. Hence we must have $\left.r_{3}=a_{2}\right\urcorner \cdot a_{3}$.

Moreover, terms $\left(a_{1}\right)=\operatorname{root}\left(a_{2}\right)=\operatorname{root}\left(a_{1}+a_{2}\right)$ also follows from Lemma 6.6 and therefore $a \in P_{1} \unrhd\left(P_{2} \bowtie P_{3}\right)$. The reverse inclusion is proved analogously.

Page 11 Lemma 7.5 needs to be discarded.

Page 11 In the proof of Lemma 7.5, after the first paragraph add:

An analogous calculation shows that $a_{1}+a_{2}$ has maximal in-degree 1 .

Lemma 7.9 (p. 12) In line 4 k needs to be replaced by j.

In the proof of Lemma 7.9 the third and fourth paragraphs should read as follows:

Further, we know $\operatorname{root}\left(a_{1}\right), \operatorname{root}\left(a_{3}\right) \neq 0$ by definition. Assume $a_{3} \neq \square$, thus $\operatorname{root}\left(a_{3}\right) \leq \Gamma_{a}$ and $\operatorname{root}\left(a_{3}\right) \neq \square$. Then $a_{1} \circledast a_{3} \Rightarrow \operatorname{root}\left(a_{1}\right) \cdot \operatorname{root}\left(a_{3}\right)=0$, Lemma 6.2 and assumptions imply $\left(\operatorname{root}\left(a_{1}\right) \mapsto \operatorname{root}\left(a_{3}\right)\right) \cdot{ }^{\top}\left\ulcorner_{a_{3}}=\operatorname{root}\left(a_{3}\right)\right.$. Moreover, $\left\ulcorner\left(\operatorname{root}\left(a_{1}\right) \mapsto \operatorname{root}\left(a_{3}\right)\right) \cdot \overrightarrow{a_{3}}=\operatorname{root}\left(a_{1}\right) \cdot \overrightarrow{a_{3}} \leq\left\ulcorner a_{1} \cdot \overrightarrow{a_{3}}=0\right.\right.$ and $\left.\left(\operatorname{root}\left(a_{1}\right) \mapsto \operatorname{root}\left(a_{3}\right)\right) \cdot a_{3}\right\urcorner=\operatorname{root}\left(a_{3}\right) \cdot a_{3} \backslash 0$. Finally, by Lemma 7.3 we have $\left(\operatorname{root}\left(a_{1}\right) \mapsto \operatorname{root}\left(a_{3}\right)\right)+a_{3} \in l_{-} \operatorname{context}(p) \bowtie R(q)$.

It remains to show $\left(\operatorname{root}\left(a_{1}\right) \mapsto \operatorname{root}\left(a_{3}\right)\right) \circledast a_{2}$ and $a_{3} \circledast a_{2}$. The latter follows from commutativity of $\circledast$ while the former resolves to $\operatorname{root}\left(a_{1}\right) \cdot \overline{a_{2}}=0$ and $\operatorname{root}\left(a_{3}\right) \cdot \overrightarrow{a_{2}}=0$ by Lemma 6.2 . Hence, $\operatorname{root}\left(a_{1}\right) \cdot \overrightarrow{a_{2}}=0$ by $a_{1} \triangleright a_{2}$. Similarly, $\operatorname{root}\left(a_{3}\right) \cdot \overrightarrow{a_{2}} \leq 0$ by assumptions. 
Page 14 In line 11 the trees in question should read $T_{l}, T_{k}, T_{r}$. The correctness proof should read as follows:

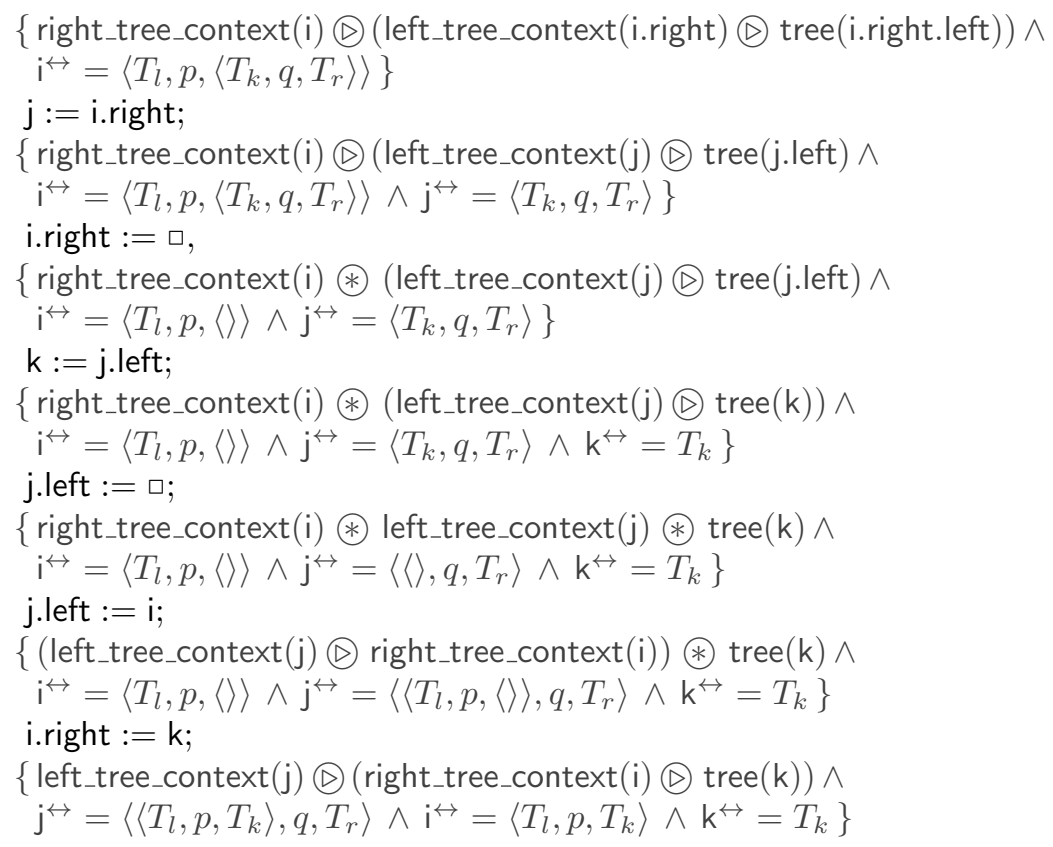

The optimised program should read as follows:

$$
\begin{aligned}
\left\{\mathrm{i} \leftrightarrow=\left\langle T_{l}, p,\left\langle T_{k}, q, T_{r}\right\rangle\right\rangle\right\} & \mathrm{j}:=\mathrm{i} . \text { right } \\
& \text { i.right }:=\mathrm{j} . \text { left } ; \\
& \text { j.left }:=\mathrm{i} ; \\
\left\{\mathrm{j} \leftrightarrow=\left\langle\left\langle T_{l}, p, T_{k}\right\rangle, q, T_{r}\right\rangle\right\} &
\end{aligned}
$$

